\title{
La PAC en débat : regards sur nos partenaires européens
}

\author{
Mise en œuvre et premiers impacts de la réforme de 2003 \\ et du système d'aides simplifié dans quelques États membres
}

\author{
Bénédicte CARLOTTI \\ Pluriagri, \\ 43-45, rue de Naples \\ 75008 Paris, France \\ <carlotti@pluriagri.fr>
}

\begin{abstract}
While the debate about the health check of the Common Agricultural Policy is starting, what lessons can be drawn from the implementation of the previous 2003 CAP reform in the different UE Member states? The EU Commission granted a large flexibility to EU-15 Member states to implement the Single Payment Scheme (SPS). Pluriagri, an association created by the French producers of arable crops and Crédit Agricole to achieve prospective analysis on agriculture and agricultural policies, made several interviews during the year 2007 in selected Member states to enlighten the first impacts of these various choices. An additional analysis of the first consequences of the accession on the agriculture of selected new Members is given (Poland, Hungary). This article points out that the effect of the implementation of the 2003 CAP reform started to be felt in the first year of implementation (2005/2006), then was rapidly masked by the improvement in agricultural markets and the bearing effect of bio energies. A question remains open: what would have been the situation if prices had remained low?
\end{abstract}

Key words: common agricultural policy, single payment scheme, decoupling, regionalized models
Alors que les débats sur la proposition de la Commission européenne pour le bilan de santé de la Politique agricole commune (PAC) ont commencé, quels enseignements peut-on tirer des choix de nos partenaires européens dans l'application de la réforme précédente de 2003 ?

Cet article reprend les présentations faites lors de la Journée Prospective Pluriagri qui s'est tenue le 18 décembre 2007. II revient sur les modalités d'application adoptées par plusieurs États membres et fait le point sur les principaux impacts que l'on a pu identifier un à deux ans après la mise en œuvre de la réforme.

La spécificité de la réforme de la PAC de 2003 résidait dans l'introduction du régime de paiement unique (RPU, SPS en anglais), c'est-à-dire dans la suppression des liens entre la production et les subventions. Les paiements ainsi découplés assurent un soutien au revenu des agriculteurs tout en permettant I'orientation de la production en fonction de la demande du marché; avec pour conséquence, le cas échéant, un simple entretien des terres sans acte de production.

La réforme de 2003 visait également à promouvoir une agriculture écologiquement et économiquement durable à travers la conditionnalité des aides, et leur modulation - rendue obligatoire - afin de financer des mesures supplémentaires de développement rural.

La Commission européenne a concédé une large subsidiarité aux Etats membres de I'UE-15 dans l'application du régime de paiement unique. Cette flexibilité s'exerçant essentiellement sur trois aspects de sa mise en œuvre : le calen- drier d'introduction du RPU, les modalités de calcul des droits à paiement unique et les options de découplage. Ainsi :

- les États membres avaient la latitude d'introduire le RPU à partir du $1^{\mathrm{er}}$ janvier 2005 ou de le différer jusqu'au $1^{\mathrm{er}}$ janvier 2007 au plus tard ; - les droits à paiement unique pouvaient être calculés sur une base individuelle historique dans laquelle chaque agriculteur obtient des droits correspondant aux paiements qu'il recevait pendant la période de référence 20022004, ou bien selon une approche régionale (montant forfaitaire ou modèle hybride), approche qui implique une certaine redistribution des paiements entre agriculteurs;

- les États membres étaient autorisés à conserver une certaine proportion d'aides couplées (découplage partiel) pour des productions pour lesquelles le passage à un découplage total pouvait faire craindre des perturbations des marchés agricoles ou provoquer l'abandon de la production ;

- enfin, dans le cadre de l'article 69 du règlement 1782/2003, les États membres pouvaient, par un prélèvement atteignant jusqu'à $10 \%$ des aides du ${ }^{\text {er }}$ pilier, accorder des paiements supplémentaires pour encourager la protection et l'amélioration de l'environnement ou bien afin d'améliorer la qualité et la commercialisation des produits agricoles.

Les États membres ont largement utilisé la gamme des options possibles. Nous retiendrons dans cet article le cas de quatre d'entre eux: I'Allemagne, I'Espagne, I'Italie et le Royaume-Uni (plus précisément l'Angleterre), dont les choix sont résumés sur la figure 1.
Pluriagri a réalisé une série d'entretiens au cours de l'année 2007 dans les États membres cités, auprès d'experts de la profession agricole, des filières et de l'administration, afin de cerner les premiers impacts de ces choix différenciés, en privilégiant deux angles qui seront successivement abordés :

- les conséquences du découplage total sur les assolements, la production et les filières, particulièrement illustrées par les cas de I'Italie et de I'Espagne ;

- l'effet de redistribution des soutiens par les modèles régionalisés à partir du cas de l'Allemagne et de l'Angleterre.

Dans le contexte de réflexion sur le bilan de santé de la PAC, cette synthèse sera complétée par une analyse des premières conséquences de l'adhésion à l'Union européenne sur l'agriculture des nouveaux États membres, en particulier la Pologne et la Hongrie.

\section{Les conséquences du découplage total sur la production et les filières}

Une chute drastique des surfaces en blé dur en Italie...

La réforme de la PAC est entrée en vigueur en Italie dès la campagne 2005 selon un modèle de découplage total fondé sur des références historiques individuelles pour les grandes cultures et les productions animales. L'Italie a choisi d'appliquer l'article 69 afin d'encourager des pratiques présentant un intérêt particulier 


\section{Modalités de calcul du DPU}

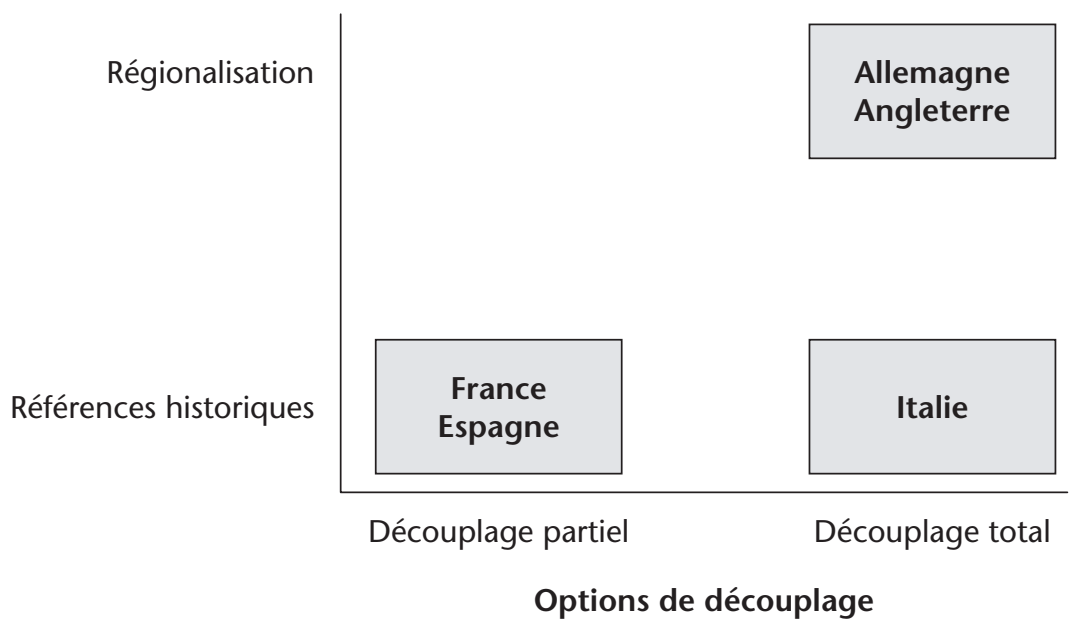

Figure 1. Choix retenus par les États membres pour la mise en oeuvre de la réforme de la PAC 2003. D'après /C. Kroll

vis-à-vis de l'environnement ou selon des critères de qualité. Mais compte tenu du faible niveau d'exigence des conditions d'admissibilité, l'application de l'article 69 n'a permis en définitive qu'un saupoudrage de compléments d'aides.

Dès 2005, les experts italiens dans leur ensemble anticipaient un impact majeur de la réforme sur les surfaces en céréales, et plus particulièrement en blé dur. Mais, faisant ce choix du découplage total, l'Italie s'engageait délibérément dans la voie d'une orientation de la production vers le marché et de la maximisation des aides au détriment éventuel des surfaces cultivées.

De fait, le découplage total a provoqué une chute drastique des surfaces en blé dur en Italie, dont l'estimation varie selon les sources statistiques, mais que l'on peut considérer selon I'Institut national de statistiques (ISTAT) et I'Institut de services pour les marchés agricoles et alimentaires (ISMEA) de l'ordre de - $25 \%$ des surfaces entre 2004 et 2006 (environ - $450000 \mathrm{ha}$ ).

Ce phénomène s'explique par le fait que les agriculteurs qui cultivaient le blé dur essentiellement « pour la prime » dans des zones peu favorables, voire défavorables, ont cessé de produire.

Cet abandon du blé dur n'a pas pour autant entraîné un abandon massif de surfaces cultivées, puisque seulement $1 / 3$ des parcelles abandonnées en blé dur ont été laissées en "bonnes conditions agricoles et environnementales » selon les règles de la conditionnalité. Les deux autres tiers ont été semés en cultures fourragères, blé tendre ou tournesol. Fait intéressant et important, la baisse des surfaces de blé dur de $25 \%$ n'a pas provoqué une chute proportionnelle de la production. La production s'est recentrée autour des producteurs spécialisés et des zones favorables. Elle s'est même partiellement déplacée vers le centre et le nord de l'Italie, où le blé dur s'est substitué au maïs et à la betterave dont les surfaces ont fortement chuté suite à la réforme de l'OCM sucre. En conséquence, la production s'est maintenue sensiblement au même niveau qu'au cours des années 2000 à 2003 (figure 2) et, de plus, la qualité s'est améliorée.

\section{Quelles ont été les répercussions sur la filière des pâtes alimentaires?}

Structurellement, I'Italie est importatrice nette de blé dur. La production nationale, à la fois en quantité et surtout en qualité, couvre au mieux
$75 \%$ des besoins de la transformation. Dans ce contexte, l'introduction du découplage total s'est traduite pour les fabricants de pâtes par une poursuite de l'importation. Néanmoins, la qualité de l'approvisionnement national s'est sensiblement améliorée notamment via le développement de contrats de production entre industriels et agriculteurs, pratique jusqu'alors très peu développée dans ce secteur de l'agriculture italienne.

Courant 2007, avec la situation nouvelle sur les marchés agricoles internationaux et la flambée des prix particulièrement forte pour le blé dur, les semis repartent à la hausse dans toutes les régions italiennes, illustrant l'interaction de la production avec les signaux du marché souhaitée à travers le découplage.

Avec le recul, les experts considèrent que I'introduction du découplage total est positive, qu'elle a permis de faire évoluer le secteur de production, et a surtout maximisé le soutien en évitant une déperdition d'aides comme cela a été observé en Espagne.

\section{Un découplage partiel incapable d'endiguer l'abandon de production en Espagne...}

L'application du régime de paiement unique a débuté dans toutes les communautés autonomes d'Espagne pour la récolte 2006 sur la base des références historiques individuelles des agriculteurs. Les choix du ministère de l'Agriculture pour la mise en œuvre de la réforme de la politique agricole commune ont été guidés par la volonté d'éviter au maximum l'abandon de l'activité agricole et la déprise rurale dans un pays où des zones importantes sont menacées de désertification: d'où un recouplage au maximum des possibilités (25\%) et l'application de l'article 69.

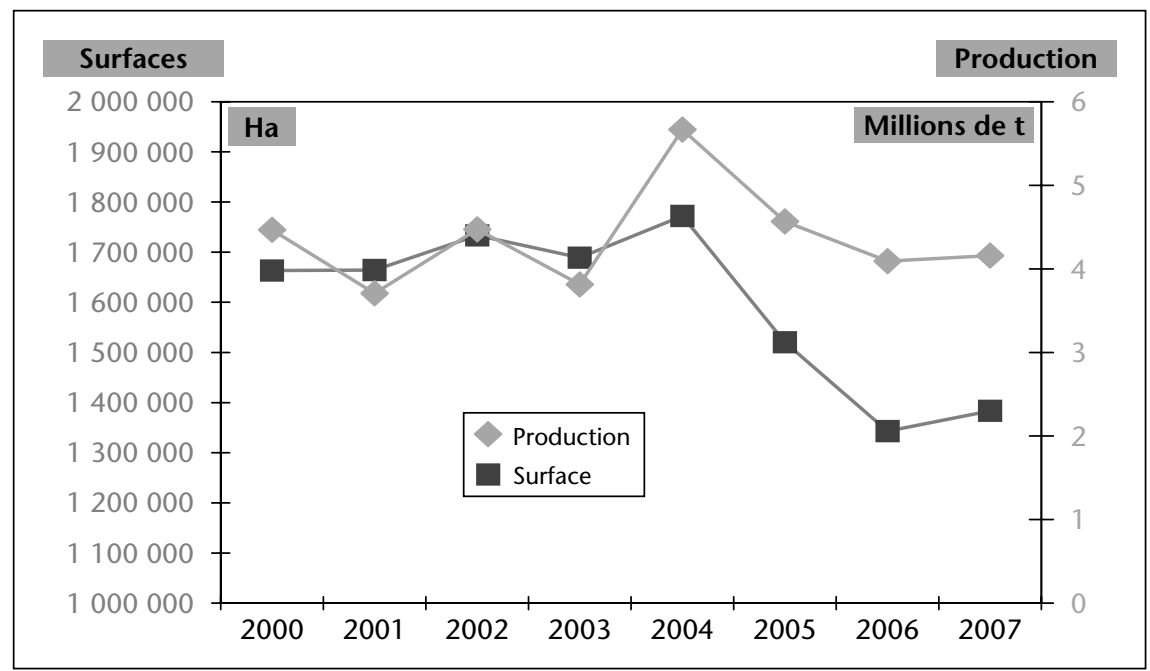

Figure 2. Évolution des surfaces cultivées et de la production de blé dur en Italie. Source : ISTAT 
Tableau 1. Évolution des surfaces cultivées en Espagne suite à l'introduction du découplage partiel. Source : Mapya.

\begin{tabular}{|c|c|c|c|c|}
\hline Source : Mapya & 2006 par rapport à 2005 & 2007 par rapport à 2005 & \multicolumn{2}{|c|}{ Cumul Ha \% de la sole correspondante } \\
\hline Total céréales & -281393 ha & -187513 ha & -468906 ha & $-7 \%$ \\
\hline dont Blé dur & -275430 ha & - 184752 ha & -460182 ha & $-45 \%$ \\
\hline Tournesol & + 111371 ha & + 7501 ha & +118872 ha & $+23,5 \%$ \\
\hline Surfaces fourragères & + 9000 ha & +70000 ha & + 79000 ha & $+9 \%$ \\
\hline Jachères ("barbechos y posios") & + 196500 ha & +148000 ha & +344500 ha & $+10 \%$ \\
\hline
\end{tabular}

Cependant, le recouplage partiel n'a pas permis d'éviter la réduction des surfaces en céréales.

La sole espagnole a diminué de presque 470000 ha de céréales (soit - $7 \%$ ) en deux campagnes par rapport aux surfaces enregistrées en 2005, dont 460000 ha de blé dur (soit - $45 \%$ ), avec des effets régionaux marqués (tableau 1).

Étant donné la faiblesse et l'irrégularité des rendements céréaliers espagnols (inférieurs à 20 quintaux par hectare dans certaines régions) et compte tenu des prix de marchés, il s'est avéré plus rentable pour les agriculteurs d'arrêter la production en recevant uniquement les $75 \%$ découplés, plutôt que d'engager des coûts pour toucher les $25 \%$ recouplés supplémentaires

Cette situation a provoqué de facto, une perte de $25 \%$ du soutien au titre du $1^{\mathrm{er}}$ pilier pour ce secteur de l'agriculture espagnole. Selon certaines études du ministère de l'Agriculture, un recouplage de l'ordre de $40 \%$ aurait été nécessaire pour éviter les abandons de production, mais le règlement communautaire ne le permettait pas.

Du tournesol et des fourrages ont remplacé le blé dur sur une partie des surfaces délaissées. Comme le montre le tableau 1, la sole de tournesol a augmenté de presque $25 \%$ en 2007 par rapport à 2005, et celle des surfaces fourragères $\mathrm{d}^{\prime}$ environ $10 \%$.

Mais, l'essentiel des surfaces abandonnées par le blé dur a été maintenu en bonnes conditions agricoles et environnementales selon les exigences de la conditionnalité et, temporairement, mises en jachère selon les pratiques agronomiques des milieux semi-arides. Ces surfaces pourraient retourner en production avec I'amélioration des marchés.

\section{Mais l'appel du marché et l'effet porteur des biocarburants renversent la tendance...}

Dans ces deux États membres, l'Italie et l'Espagne, la flambée du prix du blé dur sur le marché mondial liée à une pénurie dans l'ensemble des pays producteurs, provoque aujourd'hui une inversion de la tendance avec la reprise des semis de blé dur pour la récolte 2008.
Autre production, autre contexte. Contrairement à ce qui était craint, le seigle n'a pas été le grand perdant de l'introduction du découplage total en Allemagne et les surfaces à faible potentiel des Länder de l'Est n'ont pas été abandonnées.

En effet, le seigle est majoritairement cultivé sur les sols sableux à bas potentiels où il existe peu d'alternatives culturales. L'arrêt de l'intervention sur le seigle et la faiblesse du prix sur les marchés traditionnels de cette céréale avaient déterminé une tendance à la baisse continue des surfaces en Allemagne $(-25 \%$ en moyenne sur l'ensemble du pays entre 2000 et 2004). L'introduction du découplage total et l'absence d'alternative culturale laissaient supposer un déclin encore plus marqué de cette culture.

Si bien que dans ces Länder marqués par un très fort chômage rural ( $40 \%)$, on a observé une dynamique conduite par des industriels et des organismes de stockeurs pour multiplier la recherche et le développement de nouveaux débouchés pour le seigle (bioéthanol), et plus largement, pour l'ensemble des bioénergies.

En définitive, malgré le découplage total, la tendance s'est inversée et les surfaces en seigle ont progressé de $9 \%$ entre 2004 et 2007 grâce à la bonne tenue du marché du seigle sur ces débouchés traditionnels, passant de 70 à $240 € / t$ et à l'émergence d'une demande en seigle et autres céréales pour l'approvisionnement d'unités de production de bioéthanol.

Le « boom » du biogaz a provoqué un accroissement de $18 \%$ des surfaces en maïs. Avec l'effet porteur du biodiesel les surfaces en colza ont continué d'augmenter (tableau 2).

\section{L'effet de redistribution des soutiens par les modèles régionalisés}

Une chronologie vers une aide unique par hectare dans chaque Land allemand...

L'Allemagne a saisi I'occasion de l'accord sur la réforme de la politique agricole commune en 2003 pour modifier profondément son système de soutien à l'agriculture. Elle a en effet opté pour un découplage total des aides directes dès 2005 et leur régionalisation progressive dans le cadre du Kombimodell (Encadré 1, figure 3). Au terme d'une période de transition conjuguant redistribution des paiements directs (à travers la composante régionale par hectare) et maintien d'une partie du soutien calculé sur la base des références individuelles de production (à travers le «top up "), ce dispositif aboutira, en 2013, au versement d'une prime unique par hectare à tous les agriculteurs d'un même Land, d'un montant identique quelle que soit l'utilisation du sol. Le niveau de la prime unique en 2013 sera différent dans chacun des treize Länder.

Le Kombimodell traduisait la volonté de la coalition de gauche, alors au pouvoir, de répartir autrement le soutien à l'agriculture, au nom $d^{\prime}$ une plus grande équité et $d$ 'une moindre pression sur l'environnement. De fait, cette politique entraînera une redistribution plus ou moins forte du soutien selon les systèmes de production et les régions, avec des perdants et des gagnants. Que pouvait-on dire en 2007 des premiers impacts de ce modèle?

Même si la phase majeure du processus de redistribution des aides directes ne débutera qu'à partir de 2010 avec le démantèlement des top ups, dès à présent et jusqu'en 2009, un

Tableau 2. Evolution des surfaces cultivées en Allemagne suite à l'introduction du découplage partiel. Source : ZMP.

\begin{tabular}{|lccc|}
\hline & $\begin{array}{c}\text { Surfaces en 2004 avant } \\
\text { découplage total }\end{array}$ & Surfaces en 2007 & Accroissement \\
\hline Seigle & $624945 \mathrm{ha}$ & $684000 \mathrm{ha}$ & $+9 \%$ \\
Maïs ensilage & $1248468 \mathrm{ha}$ & $1475000 \mathrm{ha}$ & $+18 \%$ \\
Colza & $1279000 \mathrm{ha}$ & $1546000 \mathrm{ha}$ & $+21 \%$ \\
\hline
\end{tabular}




\section{Encadré 1}

Le Kombimodell allemand : vers une aide unique par hectare dans chaque Land

$1^{\text {re }}$ période : 2005 à 2009 - Chaque agriculteur allemand s'est vu attribuer 3 types de droits à primes :

Droit terres arables, comprenant la composante régionale définie par Land plus éventuellement la composante individuelle historique dite « top up » si I'agriculteur avait des références en production animale (lait, viande bovine, brebis), en pomme de terre fécule ou tabac sur la période 2002-2004.

Certains droits « terres arables » sont agrées fruits et légumes c'est-à-dire qu'ils ouvrent à partir de 2005 droit à l'aide sur une parcelle cultivée en fruits et légumes. Ils sont attribués à l'agriculteur au prorata de la surface en fruits et légumes qu'il cultivait durant la campagne 2003.

Sont considérés comme fruits et légumes, les espèces à destination du marché du frais ou de la transformation, y compris les pommes de terre, à l'exception des pommes de terre fécule et des cultures permanentes (arboriculture).

Ce droit est activable sur toutes les surfaces arables de l'exploitation y compris les surfaces en betterave sucrière, et sur les surfaces en fruits et légumes dans les limites des surfaces 2003 déclarées.

Droit jachère, comprenant uniquement la composante régionale définie par Land pour les terres arables, pas de composante individuelle. Ce droit est activé spécifiquement sur les surfaces en jachère.

Droit STH, comprenant la composante régionale définie par Land pour les STH (surfaces toujours en herbe) et éventuellement la composante individuelle historique dite « top up » si l'agriculteur avait des références en production animale (lait, viande bovine, brebis), en pomme de terre fécule ou tabac sur la période 2002-2004.

Ce droit est activable sur toutes les surfaces en STH, qui deviennent primées dans le Kombimodell alors qu'elles ne l'étaient pas jusqu'alors.

Le montant des droits restera identique sur la période 2005 à 2009 inclus.

À l'intérieur d'un Land, l'élément de variation entre le montant des droits à primes d'agriculteurs voisins réside uniquement dans l'existence ou non d'un top up individuel, et son niveau.

$2^{\mathrm{e}}$ période : 2010 à 2012 - convergence progressive vers une prime régionale unique

L'écart entre le montant de chaque droit à prime et la prime unique régionale objectif 2013 est progressivement réduit.

La redistribution s'opère par le transfert progressif des montants perçus au titre de la composante historique individuelle « top up » vers la composante régionale.

$3^{\mathrm{e}}$ étape - 2013 - une prime unique par Land

Le modèle devient totalement régionalisé : tous les agriculteurs d'un Land reçoivent la même prime par hectare, et ce, quel que soit le type de parcelles (terres

arables, jachère, STH).

premier niveau de redistribution a été effectué qui a orienté à la baisse les aides perçues par les exploitations spécialisées en céréales et oléoprotéagineux (SCOP) et les élevages intensifs de viande bovine.

En revanche, le soutien perçu par les producteurs de betteraves à sucre, de pommes de terre et de fruits et légumes de plein champ est en hausse puisque la quasi-totalité de leur superficie agricole utilisée est devenue éligible, de même pour les éleveurs de bovins (viande et lait) extensifs, dont la SAU est composée pour une large part de prairies permanentes désormais primées.

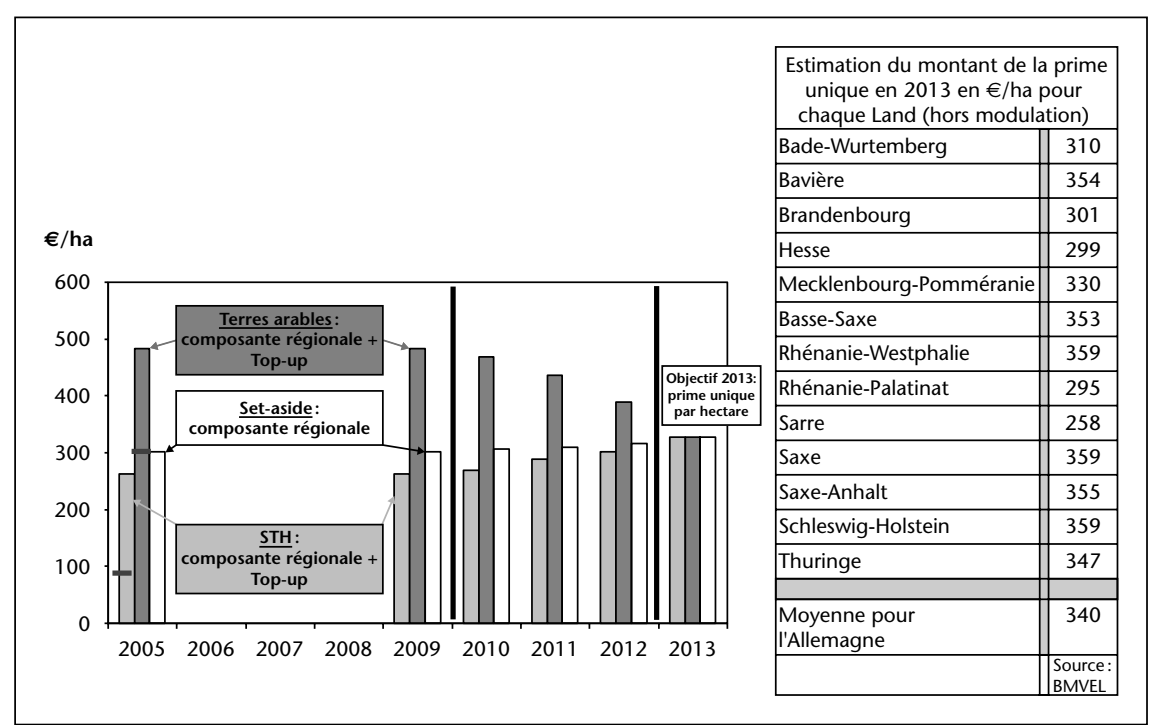

Figure 3. Redistribution progressive des top ups vers I'aide régionale unique en 2013.
Par ailleurs, les producteurs de betteraves à sucre et les éleveurs laitiers bénéficient de top ups élevés correspondant à la prime laitière et à la prime compensatoire betterave dans le cadre de la réforme des OCM. Ces deux systèmes, aujourd'hui « gagnants » seront les plus touchés par la redistribution des aides liée au démantèlement des « top ups » en 2010.

Selon une communication du DBV, l'effet «perdant/gagnant» s'est en partie observé dans les comptabilités des exploitations la première année de mise en œuvre du Kombimodell. Cependant, la réaction des agriculteurs allemands concernant la réforme de la PAC s'est focalisée bien davantage sur la complexité de la mise en œuvre de la conditionnalité que sur la redistribution du soutien.

Puis, au cours de la deuxième année d'application (2006/2007), la bonne santé des marchés agricoles a engendré une hausse des revenus qui a masqué la redistribution des aides entre systèmes de production.

\section{Des agriculteurs britanniques plus sensibles à la modulation qu'à la régionalisation...}

Chacune des nations du Royaume-Uni a choisi ses propres modalités de mise en œuvre de la réforme de 2003. 
L'Angleterre a opté pour une application dès 2005 de droits à paiements totalement découplés calculés selon un modèle régional de type hybride dynamique. Le territoire anglais a été divisé en trois zones de potentialités différentes: les zones de landes (Moorlands), les autres zones défavorisées (disanvantaged areas), et enfin, une troisième zone constituée du reste de l'Angleterre.

Au sein de chaque zone, les droits à paiement unique perçus par un agriculteur intègrent une part de références historiques individuelles de l'agriculteur sur la période 2000-2002 et une part régionale dont les proportions relatives évoluent sur 8 ans vers une aide purement régionale en 2012 (figure 4).

À la fin de la période de transition, les aides uniques à la surface sont estimées à $60 € /$ ha dans la zone dite des Moorlands et à $180 € /$ ha dans les autres zones défavorisées. Dans le reste de I'Angleterre, où se situe l'essentiel des exploitations de grandes cultures, I'aide unique s'élèverait à $330 € /$ ha.

Le gouvernement anglais a justifié ce choix en argumentant qu'une approche fondée uniquement sur les références historiques ne serait pas pérenne. L'opinion publique ne comprenant pas au bout de quelques années que les agriculteurs perçoivent des aides sur la base de leur activité passée alors que le contexte économique et de la politique agricole ont évolué. Admettant que le passage immédiat à un tel dispositif aurait eu un effet de redistribution des aides de la PAC massif et potentiellement déstabilisateur pour certaines filières, une période de transition de huit ans avec une accélération à compter de la troisième année a été retenue pour permettre à l'agriculture anglaise de s'adapter.

L'effet de redistribution attendu devrait s'exercer en faveur de la filière ovine et des exploitations de grandes cultures mixtes dont les surfaces en betteraves, pommes de terre, etc. deviennent éligibles. S'agissant des exploitations spécialisées grandes cultures, les aides perçues en 2012 seraient réduites de $30 \%$ par rapport au soutien de 2004 .

Quels étaient en 2007 les premiers effets?

Le mécontentement des producteurs s'est surtout cristallisé autour des retards de paiement des aides plus que sur leur mode de calcul. L'effet de redistribution du soutien par le modèle régionalisé est encore faible puisqu'en 2007 l'aide restait calculée à $70 \%$ sur les références historiques de I'agriculteur. En revanche, l'effet de la modulation est net. L'Angleterre applique une modulation nationale volontaire, dont le taux était fixé à $12 \%$ en 2007. Les aides du premier pilier ont ainsi été réduites d'un montant total de $17 \%$ (5\% au titre de la modulation obligatoire et $12 \%$ au titre de la modulation volontaire) pour financer les programmes de développement rural, pour l'essentiel à des fins environnementales.

Dans leur ensemble, les agriculteurs britanniques disent « jouer le jeu »... car les programmes environnementaux ne sont - pas encore trop contraignants et leur permettent de recouvrer une partie des aides perdues sur le $1^{\mathrm{er}}$ pilier au travers des programmes agrienvironnementaux auxquels ils participent. Mais les agriculteurs sont très inquiets du renforcement probable des contraintes exigées par ces programmes à l'avenir et de la distorsion de concurrence qu'introduit la modulation volontaire.

S'agissant des éleveurs, l'impact négatif des épizooties prime largement sur les effets de la réforme de la PAC ou de la modulation.

\section{Les conséquences de l'intégration à l'UE sur l'agriculture des nouveaux États membres}

\section{Un système d'aide simplifié transitoire pour les nouveaux États membres..}

La Hongrie et la Pologne ont adopté lors de leur adhésion à I'Union européenne le régime de paiement à la surface (RPUS ou SAPS en

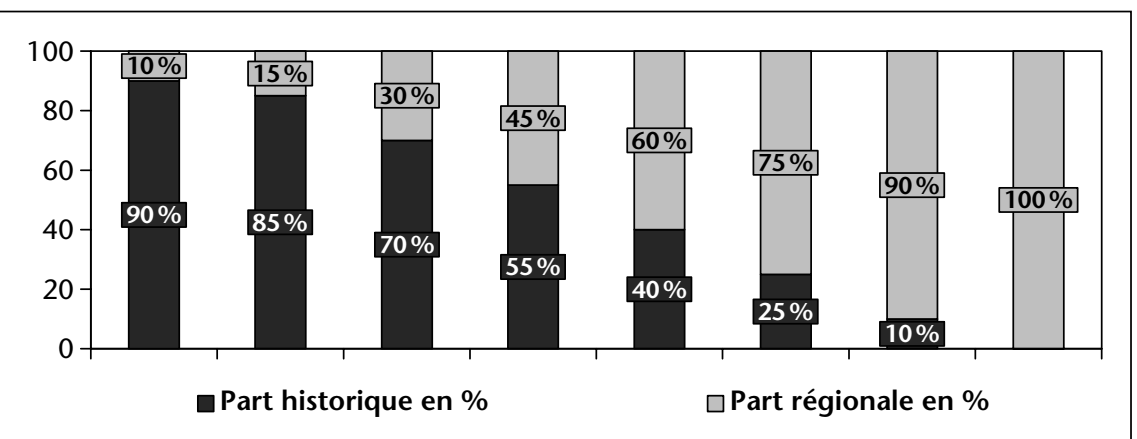

Figure 4. Évolution relative de composantes historique et régionale dans le modèle hybride dynamique anglais. Source Defra. anglais), système d'aides directes simplifié introduit en 2004 pour une durée transitoire de trois ans puis reconduit deux ans. Il est donc en vigueur jusqu'au $1^{\text {er }}$ janvier 2009.

Ce système octroie une aide communautaire sous la forme d'une aide directe à l'hectare, découplée, dont le montant augmente graduellement sur la période 2004-2013 de $25 \%$ à $100 \%$ du plafond national résultant des accords d'adhésion (respectivement 1,309 milliard d'euros en 2013 pour la Hongrie, 3 milliards d'euros pour la Pologne).

Conformément aux textes, les gouvernements nationaux majorent l'aide SAPS par un complément pris sur le budget national dit « top up » ne pouvant excéder annuellement $30 \%$ du plafond national fixé pour l'Etat membre.

Le total " aide communautaire + top up » ne peut lui-même dépasser un pourcentage prédéfini du plafond national (figure 5).

L'Etat membre peut par les modalités du « top up " (nature des mesures et degré de découplage) orienter les productions agricoles, tout en restant dans le cadre des OCM.

La Hongrie a fait le choix jusqu'en 2006 de coupler tous les « top ups » à la production en ciblant particulièrement le secteur de l'élevage bovin affaibli par la période de transition économique. Puis, en 2007, à la demande de la Commission, le découplage total a été introduit pour le lait et les grandes cultures, mais le couplage partiel toutefois maintenu pour l'élevage bovin viande et le tabac.

La Pologne, quant à elle, a pris l'option de verser les « top ups » sous forme d'un paiement unique totalement découplé, sur toutes les surfaces éligibles (SCOP, surfaces fourragères, prairies permanentes, cultures énergétiques) dès 2004.

\section{Quels sont, quatre ans après}

I'adhésion, les principaux impacts

du système d'aides directes sur ces agricultures?

La restructuration de l'agriculture hongroise s'est accélérée au détriment des petites fermes d'élevage : en effet, malgré le ciblage des « top ups ", l'érosion de l'élevage bovin s'est poursuivie et les importants retards de paiement des aides directes durant la première année ont particulièrement fragilisé les petites exploitations. De surcroît, les secteurs porcins et avicoles soutenus par la politique agricole nationale avant l'adhésion ne perçoivent désormais plus d'aides.

En revanche, les aides directes ont eu un impact très positif sur les exploitations de grandes cultures de Hongrie, comme d'ailleurs de Pologne. Ce soutien a encouragé la modernisation des exploitations professionnelles, même s'il a induit indirectement une augmen- 


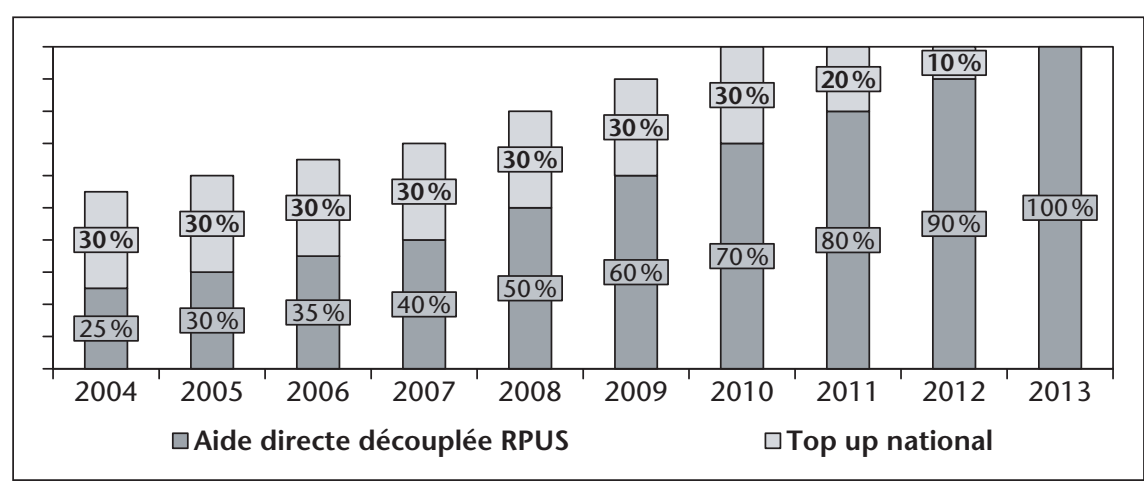

Figure 5. «Phasing-in » des aides du $1^{\text {er }}$ pilier dans le système RPUS.

tation du coût des intrants et du prix de la terre et des fermages.

En Pologne, environ la moitié des exploitations touchant les aides directes sont des exploitations de semi-subsistance. La légitimité du versement des aides à cette catégorie d'exploitations n'est généralement pas remise en cause, bien que ce soutien freine la restructuration de l'agriculture. Les subventions sont considérées comme des aides sociales permettant notamment aux ménages les plus pauvres d'assurer I'instruction des jeunes générations qui, selon toute vraisemblance, quitteront à terme le secteur agricole.

\section{Des stratégies distinctes}

mais une préoccupation commune aux nouveaux États membres...

Le système d'aides transitoire RPUS a été mis en place dans la plupart des nouveaux États membres au $1^{\mathrm{er}}$ mai 2004, pour trois années (20042005-2006) renouvelable pour deux ans (2007-2008). II prendra donc fin au $1^{\mathrm{er}}$ janvier 2009, date à laquelle les États membres de I'UE-10 auront le choix :

- soit, de prolonger leur régime actuel de paiement unique à la surface (RPUS) pour deux années supplémentaires, c'est-à-dire jusqu'au $1^{\mathrm{er}}$ janvier 2011 ;

- soit, de passer dès le $1^{\text {er }}$ janvier 2009 au régime de paiement unique (RPU) en vigueur dans les anciens États membres avec les options de découplage total versus recouplage partiel, de références historiques versus modèle régionalisé.

Le choix de la Hongrie est de rejoindre au plus vite le système d'aides des anciens États membres. Les arguments avancés ne portent pas tant sur la nature respective des deux systèmes, que sur la stratégie de s'inscrire dans le même schéma que les États de I'UE-15. La Hongrie, convaincue de son potentiel agricole, notamment grâce à la qualité de ses sols, souhaite peser sur les choix de politique agricole de
l'Union à parité avec les anciens États membres.

Contrairement à la Hongrie, la Pologne souhaite prolonger le régime de paiement unique à la surface (RPUS) jusqu'en 2013. Cela suppose qu'elle obtienne de l'UE l'autorisation de prolonger une nouvelle fois - pour deux ans ce système, dont la date limite d'application est actuellement fixée au $1^{\text {er }}$ janvier 2011. Les experts considèrent qu'il serait beaucoup trop compliqué et coûteux d'introduire le RPU (SPS) en Pologne, car l'extrême morcellement des terres (30 millions de parcelles) compliquerait énormément la gestion et le contrôle de I'activation des droits à aide. Ils sont également apparus très réticents, par principe, à l'instauration d'un marché de ces droits.

En dépit de ces différences de stratégie, la Pologne comme la Hongrie dénoncent l'écart (€/ha). qui subsistera en 2013 entre le niveau des paiements directs versés aux agriculteurs des nouveaux États membres (UE-10), lorsqu'ils toucheront l'aide communautaire à taux plein, et le montant des paiements octroyés dans les anciens États membres (UE-15).

Cet écart serait en moyenne de $49 €$ par hectare éligible, soit $16 \%$ (figure 6). En Pologne, qui recevra moins que la moyenne des nouveaux États membres, I'aide par hectare éligible serait inférieure de $103 €(34 \%)$ à celle versée dans I'UE-15. C'est en Hongrie que la différence serait la moins forte.

Si l'on détaillait la comparaison avec les anciens États membres, dont la France, qui touchent une aide supérieure à la moyenne de I'UE-15, les écarts d'aides par hectare entre nouveaux et anciens États membres seraient encore plus importants.

Les différences dans le niveau des aides par hectare entre pays proviennent essentiellement des rendements de référence pris en compte pour le calcul des enveloppes d'aides. Certains experts des nouveaux États membres considèrent que ces rendements, qui reflètent les niveaux historiques de performance des agricultures des membres, ne constituent pas une base pertinente pour le calcul du soutien dans la mesure où tous les producteurs européens sont confrontés, quel que soit le pays, au même univers concurrentiel.

Ils voudraient ainsi extrapoler entre États membres le raisonnement tenu par la Commission européenne dans son projet de communication sur le bilan de santé de la PAC, selon lequel il sera de plus en plus difficile de justifier, par

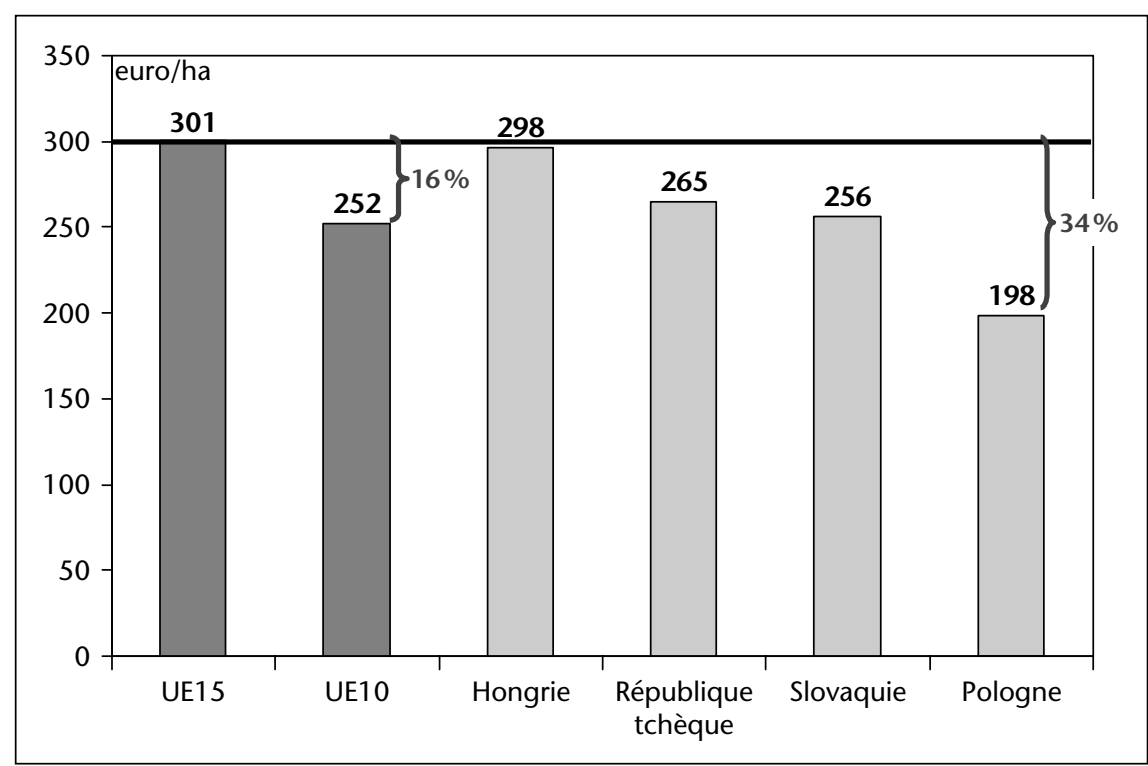

Figure 6. Paiement direct moyen perçu par hectare éligible dans les anciens et les nouveaux États membres en 2013 
des raisons historiques, les différences de montant des aides existant au sein de chaque État membre.

Cet argument, relatif uniquement aux aides du premier pilier de la PAC, ne tient pas compte de I'ampleur du soutien reçu par les pays d'Europe centrale et orientale au titre du deuxième pilier. À titre indicatif, les fonds communautaires prévus en faveur du développement rural pour la période 2007-2013 s'élèvent à 13,2 milliards d'euros pour la Pologne et 3,8 milliards d'euros pour la Hongrie, ce qui en fait respectivement les premier et huitième bénéficiaires de l'enveloppe du Fonds européen agricole pour le développement rural (FEADER).

La préoccupation exprimée par les nouveaux États membres laisse en tout cas supposer que l'équité de la répartition des aides et l'équilibre entre les volets « marché » et « développement rural » seront au cœur des débats sur le bilan de santé de la PAC et « l'après-2013 ».

\section{Conclusion}

La série d'entretiens conduits dans six États membres permet, sans prétendre à l'exhaustivité, de tirer des enseignements sur la mise en œuvre de la réforme de la PAC de 2003 et I'introduction du régime d'aides directes dans les nouveaux entrants. Il apparaît que les diverses modalités d'application choisies selon les pays ont eu des conséquences dès la première année de mise en œuvre de la réforme (2005/2006) :

- substitution ou abandon de production, suite au découplage total ou même partiel ; - amorce de redistribution des aides du $1^{\mathrm{er}}$ pilier par les modèles régionalisés, générant perdants et gagnants.
Cependant, ces effets ont été rapidement masqués par la hausse des prix observée sur la plupart des marchés agricoles et, localement, par l'effet porteur des bioénergies.

Une question se pose : qu'en aurait-il été si les prix des grandes cultures étaient restés à un bas niveau ? Compte tenu du poids énorme des aides dans le revenu des exploitations agricoles, les réflexions en cours sur l'opportunité d'une modification des modalités du découplage et de la répartition des paiements directs ne peuvent s'affranchir de l'hypothèse d'un retournement possible des marchés, ou du moins de l'accroissement de leur volatilité. De ce fait, elles ne peuvent être traitées séparément des questions relatives au maintien ou à la réduction des instruments actuels de régulation des marchés et à la création éventuelle de nouveaux outils de gestion des risques. 